\title{
Introduction of advanced photonics research at the undergraduate level: an example from the Philippines
}

John Liwag, Kees Karremans

John Waruel Liwag, Kees Karremans, "Introduction of advanced photonics research at the undergraduate level: an example from the Philippines," Proc. SPIE 4588, Seventh International Conference on Education and Training in Optics and Photonics, (28 May 2002); doi: 10.1117/12.468754

SPIE Event: Education and Training in Optics and Photonics 2001, 2001, Singapore, Singapore 


\title{
The introduction of advanced photonics research at undergraduate level: an example from the Philippines
}

\author{
John Waruel F. Liwag ${ }^{1}$ and Kees Karremans ${ }^{2}$ \\ Optoelectronics Laboratory, Department of Physics, University of San Carlos \\ Cebu City 6000, Philippines
}

\begin{abstract}
AB STRACT
Laser cooling experiments are a good example of how the interaction between light and matter can be applied to manipulate neutral atoms. At the University of San Carlos we have constructed a magneto-optical trap (MOT) for rubidium $(\mathrm{Rb})$ atoms. The setup is based on the design of the MOT Wieman et al used in their efforts to reach BoseEinstein condensation. The use of diode lasers enables the introduction of these advanced experiments at undergraduate level. Since 1998, when the MOT became operational at USC, a great number of undergraduate students have done the experiment. Not only did they learn the basics of cooling and trapping, they also gained experience in operating lasers properly, vacuum technology, and techniques for frequency stabilization. In some cases students opted to perform spectroscopy experiments on $\mathrm{Rb}$ atoms, which could easily be done using this setup.

Of course, the presence of the MOT also gave a great boost to the research in optoelectronics, a new research direction in our department. Characterizing the MOT has resulted in several scientific papers in local physics journals as well as presentations to the annual Philippine Physics Congress. The next step is towards the realization of Bose-Einstein condensation of $\mathrm{Rb}$ in our Optoelectronics Laboratory.
\end{abstract}

Keywords: magneto-optical trap (MOT), laser cooling and trapping, spectroscopy, rubidium, Bose-Einstein condensation (BEC), University of San Carlos, diode lasers, frequency stabilization, optoelectronics

\footnotetext{
1e-mail:jwfliwag@hotmail.com

2e-mail:k_karremans@hotmail.com
} 


\section{INTRODUCTION}

The topic of cooling and trapping of neutral atoms using lasers has received quite a lot of attention over the last few years. Most noticeable was the attribution of the Nobel Prize in Physics in 1997 to $\mathrm{Chu}^{1}$, Cohen-Tannoudji ${ }^{2}$, and Phillips ${ }^{3}$ for their pioneering work in the field of laser cooling and trapping. The technique of using lasers to cool down neutral atoms has found many applications in experimental physics over the last decade. A well-known example is the application of laser cool techniques to cool down atoms to the temperature regime in which the atoms show collective behavior. The observation of this quantum mechanical phenomenon, known as Bose-Einstein condensation (BEC) ${ }^{4}$, was awarded this year's the Nobel Prize. It is clear from these examples that laser cooling is a new and exciting technique, which creates opportunities to explore new fields in physics.

The exciting developments in this research field were a strong motivation for us to initiate research in this direction. In 1997 we decided to construct a magneto-optical trap (MOT) based on the design of Wieman et al. ${ }^{5}$ Instead of using an atomic beam to load the trap, Wieman et al used a rubidium $(\mathrm{Rb})$ vapor cell, which strongly reduces the requirements for the vacuum equipment. In combination with diode lasers, a MOT can be constructed at relatively low cost (about US\$ 40,000). The small vacuum chamber and the diode lasers contribute to a setup which is relatively easy to operate. Therefore, the laser cooling experiment at the University of San Carlos does not only serve as a setup for photonics research, but that undergraduate students also use this setup in their laboratory classes and during their graduation projects. The setup provides a nice introduction of undergraduate physics major students to more advanced experiments in laser and atomic physics.

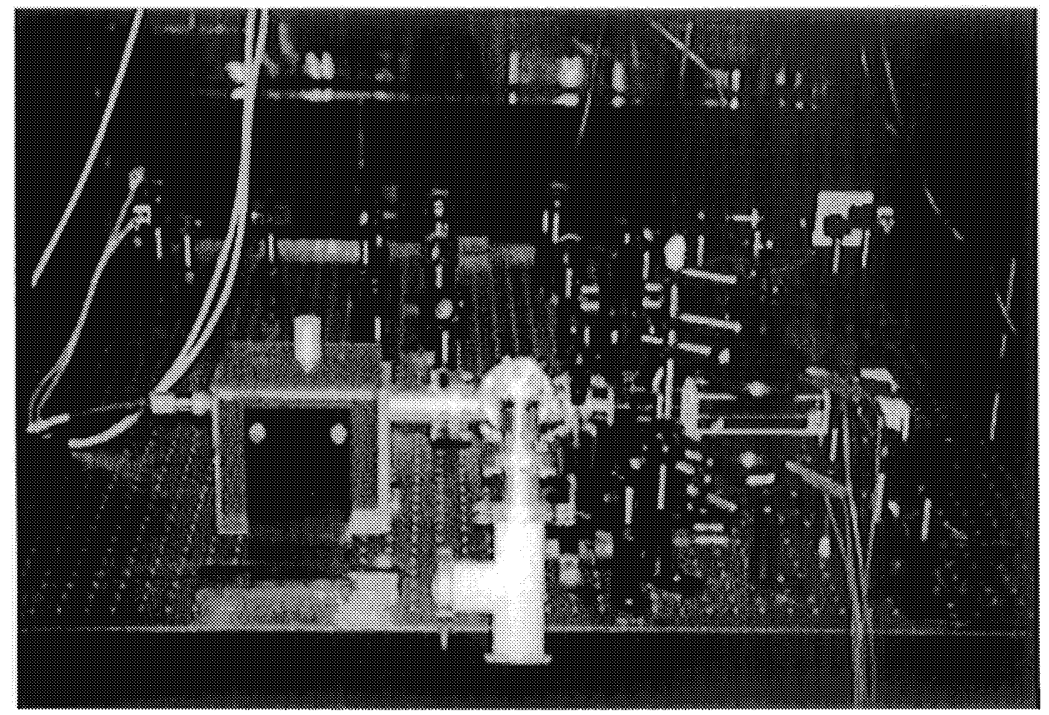

Figure 1. A photograph of the actual experimental setup of the Rb MOT at the Optoelectronics Laboratory, Department of Physics, University of San Carlos, Cebu City, Philippines.

\section{THEORY OF LASER COOLING AND TRAPPING}

In laser cooling techniques, the optical force is used to slow down atoms. The origin of this force can be understood by looking at the process of absorption and emission of photons. When the frequency of a laser is tuned to an atomic resonance, the atom can absorb a photon. In the excited state, the atom will re -emit a photon after a short period of time. For a two-level atom this process can repeat itself over and over again. Photons absorbed from the laser beam all have a well-defined propagation vector, in contrast to the emitted photons, which have a random direction. After many absorption-emission cycles, the net result is a transfer of momentum in the direction of the laser beam. 
In laser cooling, the Doppler effect is used in a clever way. By tuning the aser frequency slightly below the atomic resonance, a process known as red detuning, photons will only interact with atoms moving in the direction of the laser. Two red-detuned counterpropagating laser beams can be used to create optical molasses in one dimension. In optical molasses, atoms experience a friction force, which decreases the average velocity of the atoms in that direction. By employing this technique in three dimensions, an average velocity for $\mathrm{Rb}$ atoms of less than $2 \mathrm{~m} / \mathrm{s}$ can be reached. The corresponding minimum temperature of $144 \mu \mathrm{K}$ is known as the Doppler limit for laser cooling.

Optical molasses can be used to slow down atoms, but does not confine these atoms within a defined volume. In the MOT, the Zeeman effect is used to create a postion-dependent force. In order to drive the atoms to a confined volume, the center of the overlapping laser beams, three pairs of left- and right-circularly polarized red-detuned laser beams are required in the presence of a magnetic field that is zero at the trapping center and linearly growing outwards in all directions. This configuration is called a magneto-optical trap (MOT). Near the center of the trap the force that is exerted on the atoms in one direction $(z)$ is given by ${ }^{6}$

$$
\mathbf{F}(\mathbf{v}, z)=\frac{4 \hbar \mathbf{k}\left(2 I \Delta / I_{s} \Gamma\right)[\mathbf{k} \cdot \mathbf{v}+\beta z]}{\left(1+(2 \Delta / \Gamma)^{2}\right)^{2}}=\alpha \mathbf{v}+\kappa z
$$

where $\mathbf{k}$ is the wave vector, $\mathbf{v}$ is the velocity of the atoms, $I$ is the total intensity of the two counterpropagating laser beams, $I_{s}$ is the saturation intensity associated with the absorbing atoms, $\Gamma$ is the linewidth (FWHM) of the transition (in $2 \pi \mathrm{MHz}), \Delta$ is the detuning of the laser frequency below atomic resonance, and $\beta z$ is the linear Zeeman shift caused by the magnetic field.

Note that the force exerted on the atom can be separated into two parts: one linearly depending on the velocity of the atom and one depending on the position of the atom. The second term can be written as

$$
\mathbf{F}(z)=4 \mathrm{~kg}_{F} \mu_{B} B_{0} z \frac{I}{I_{s}} \frac{(2 \Delta / \Gamma)}{(2 \Delta / \Gamma)^{2}+\left(1+I / I_{s}\right)^{2}}
$$

where $g_{F}$ is the Landé factor associated with the transition, $\mu_{B}$ is the Bohr magneton, and $B_{0} z$ is the magnetic field at the position $z$. With a red detuning $(\Delta<0)$, this force is opposite to the sign of $z$ and increases linearly with $z$. In other words, it is a position-dependent force that drives the atoms to the center $(z=0)$ and which is zero at the center.

\section{EXPERIMENTAL SETUP}

The MOT setup consists of a glass cell maintained at low pressure by an ion pump. Rb atoms are loaded into the cell by heating the $\mathrm{Rb}$ reservoir in the system. The laser used for trapping the atoms (TUILaser ${ }^{\mathrm{TM}} \mathrm{DL} 100,50 \mathrm{~mW}$ ) is tuned to a frequency slightly lower than the resonance frequency. The rest of this beam is shaped into a circular beam and divided into three pairs of counterpropagating beams, which intersect the center of the MOT in three mutually orthogonal directions. Because of the hyperfine splitting of the ground states of $\mathrm{Rb}$, a second diode laser (SHARPTM LTO27MD, $10 \mathrm{~mW}$ ) is needed. This so-called "repumper" laser is tuned to the hyperfine transition of ${ }^{87} \mathrm{Rb}$ to insure continuous absorption-spontaneous emission cycles. It pumps electrons ending up in the lower $(F=1)$ hyperfine ground state into the $\mathrm{F}=2$ ground state, via the $\mathrm{F}=1 \rightarrow \mathrm{F}^{\prime}=2$ transition. The repumper beam is simply directed into the center of the MOT to overlap the center of the six trapping laser beams. A pair of anti-Helmholtz coils is set up such that the center of the coils coincides with the center of the glass cell, to complete the MOT configuration together with the six laser beams. 


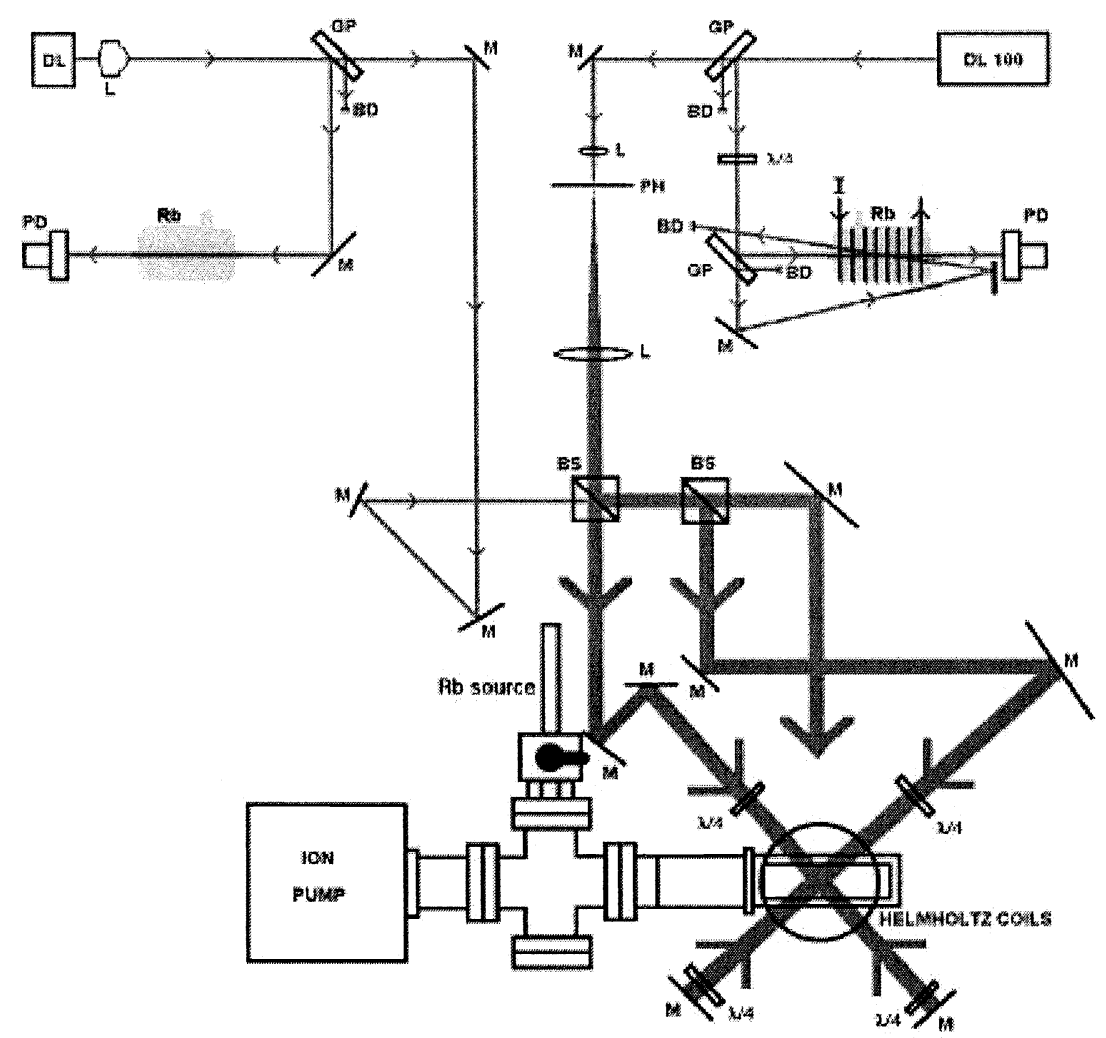

Figure 2. A schematic diagram of the experimental setup for cooling and trapping of ${ }^{87} \mathrm{Rb}$ atoms. At the top left is the repumper laser and absorption spectroscopy setup. At top right is the trapping laser and the saturation spectroscopy setup. The trapping cell with the pair of Helmholtz coils is shown in the lower part with the ion pump. DL = diode laser, DL $100=$ grating diode laser system, $\mathrm{GP}=$ glass plate, $\mathrm{M}=$ mirror, $\mathrm{L}=$ lens, $\mathrm{BD}=$ beam dump, $\mathrm{PD}=$ photodiode, $\mathrm{BS}=$ beam splitter, and $\lambda 4=$ quarterwavelength plate.

\section{SPECTROSCOPY OF RUBIDIUM ATOMS}

The $\mathrm{Rb}$ vapor cells used for laser frequency stabilization can be used to introduce spectroscopy in the laboratory classes for undergraduate students. The students learn the basic principles of laser stabilization, frequency calibration, etalons, absorption spectroscopy, and saturated absorption spectroscopy. The diode lasers used in the cooling and trapping experiments can be used to investigate the frequency range in the near infrared (IR) range. To record the absorption profile of $\mathrm{Rb}$ vapor, the temperature was kept at a constant value, while a small modulation of the injection current was used to scan the laser through the relevant frequency interval. The output of the laser diode is divided into two beams. The first beam passes through the $\mathrm{Rb}$ cell, while the second beam is used as a frequency reference. The absorption profile of the $D_{2}$ line of $R b$ is shown in the upper trace of figure 3, while the lower trace shows the frequency markers of a Fabry-Perot (FP) etalon.

Instead of using the frequency markers of the F-P etalon, some students opted to use the atomic lines for frequency calibration. The frequency calibration allows us to measure the width of the absorption dips. Making a Gaussian fit, the linewidths of the absorption dips were measured to be about $500 \mathrm{MHz}$ (FWHM). 


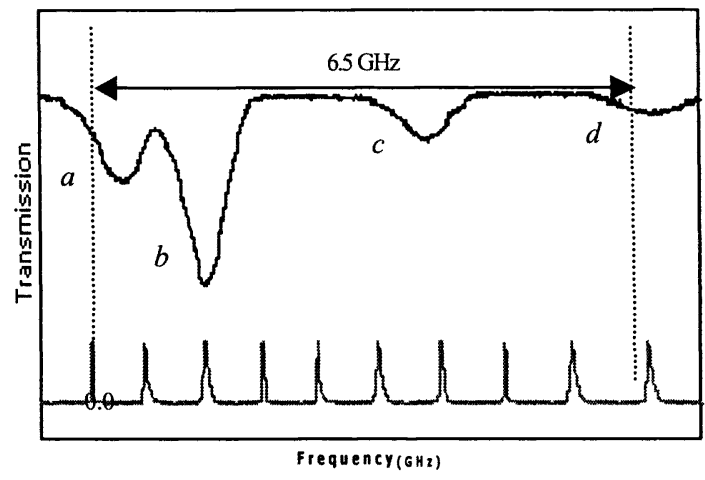

Figure 3. Absorption profile (upper trace) of the $\mathrm{D}_{2}$ line of $\mathrm{Rb}$. The peaks from the spectrum analyzer (lower trace) act as frequency markers for the range that is scanned.

The absorption peaks $a, b, c$, and $d$ of the $\mathrm{D}_{2}$ line $(780.0 \mathrm{~nm})$ of $\mathrm{Rb}$ correspond to the atomic transitions as follows:

$$
\begin{aligned}
& a:{ }^{87} \mathrm{Rb} \mathrm{5 \textrm {S } _ { 1 / 2 }}(\mathrm{F}=2) \rightarrow 5 \mathrm{P}_{3 / 2}(\mathrm{~F}=1,2,3) \\
& b:{ }^{85} \mathrm{Rb}_{5 \mathrm{~S}_{1 / 2}}(\mathrm{~F}=3) \rightarrow 5 \mathrm{P}_{3 / 2}(\mathrm{~F}=2,3,4) \\
& \text { c: }{ }^{85} \mathrm{Rb}_{5 \mathrm{~S}_{1 / 2}}(\mathrm{~F}=2) \rightarrow 5 \mathrm{P}_{3 / 2}(\mathrm{~F}=1,2,3) \\
& d:{ }^{87} \mathrm{Rb}_{5 \mathrm{~S}_{1 / 2}}(\mathrm{~F}=1) \rightarrow 5 \mathrm{P}_{3 / 2}(\mathrm{~F}=0,1,2)
\end{aligned}
$$

The most important contribution to the observed line profile is the Doppler broadening. The principle behind Doppler broadening is that the frequency of the light that the atoms will "see" is dependent on the velocity of the atoms. The Doppler width can be derived from the Maxwell-Boltzmann velocity distribution and is given by ${ }^{7}$

$$
\delta v_{D}=7.16 \times 10^{-7} v_{0} \sqrt{\frac{T}{M}}
$$

where $T$ is the temperature of the vapor cell and $M$ is the mass number of the atom. At $295 \mathrm{~K}$ the Doppler width is calculated to be around $500 \mathrm{MHz}$, in good agreement with our experimental value.

The Doppler broadening can be eliminated using saturated absorption. The improved resolution may reveal more spectroscopic details. In saturation spectroscopy, two counterpropagating light beams are used. These beams, a pump beam and a probe beam, come from the same source. The atoms that absorb light coming from the pump beam would be those that move in the direction opposite to that of the atoms absorbing the probe beam. Therefore, both beams would not be competing for absorption when the in teraction is with atoms with non-zero speed. However, the beams will compete for absorption when the interaction is with atoms with a velocity $v=0$. The effect is that a small peak, the so-called Lamb profile, will be observed at a $v=w$ in the broader absorption profile, where $v$ is the frequency of the beams and $v_{0}$ is the resonant frequency of an atomic transition. ${ }^{8}$

The increased resolution of the saturated absorption spectrum is clearly visible in figure $4 \mathrm{a}$. The $0.9 \mathrm{GHz}$ frequency scan coincides with the absorption dip $a$ in figure 3 . Instead of a single broad dip, we now observe 3 narrow lines. Only one of the peaks (the third one from the left) is identified as a Lamb profile, and is connected to the $5 \mathrm{~S}_{1 / 2}(\mathrm{~F}=2) \rightarrow$ $5 \mathrm{P}_{3 / 2}(\mathrm{~F}=3){ }^{87} \mathrm{Rb}$ transition. The Lamb profiles connected to the $5 \mathrm{~S}_{1 / 2}(\mathrm{~F}=2) \rightarrow 5 \mathrm{P}_{3 / 2}(\mathrm{~F}=1,2){ }^{87} \mathrm{Rb}$ transitions turned out to be too weak to be observed in our spectrum. The other two peaks, called "crossover" resonances, cannot directly be connected to any atomic transition. These crossovers appear exactly in between the frequency of two atomic transitions. 
The Lamb dip is independent of the Maxwell-Boltzmann distribution of the atoms. Thus, the linewidth is reduced to the order of the natural linewidth $\Gamma=5.9 \mathrm{MHz}$ of the $\mathrm{Rb}$ atomic transition. The linewidth of the Lamb profile is given by

$$
\delta v_{L P}=\Gamma \sqrt{1+\frac{I}{I_{S}}}
$$

where $I$ is the intensity of the beam passing through the vapor cell and $I_{\mathrm{S}}$ is the saturation intensity associated with the absorbing atoms.

The experimental setup can also be used to investigate the Zeeman effect. For the MOT a solenoid has been used to obtain a red detuning. In our undergraduate experiment we vary the magnetic field and record the spectrum. A spectrum recorded at the magnetic field strength of 25 Gauss is shown in Fig. 5b. The shift of the position of the peaks as a result of the Zeeman effect can be clearly observed from this graph.

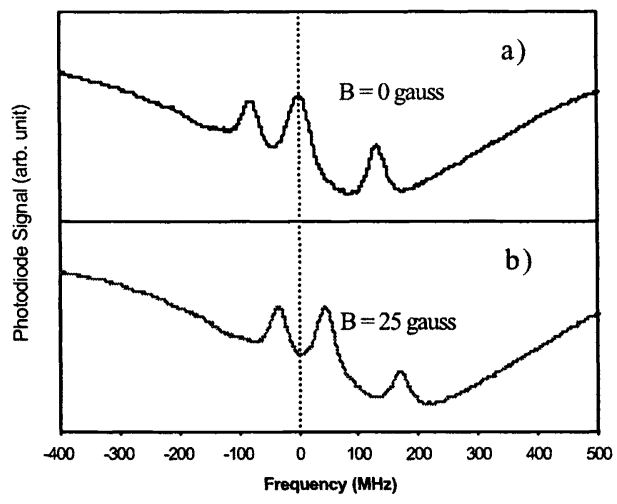

Figure 4. Atomic spectra obtained from Doppler-free saturation spectroscopy. The upper trace, a) is the spectrum serving as the frequency reference and the lower trace, $b$ ) is the spectrum of $R b$ vapor placed in a magnetic field.

\section{CHARACTERIZATION OF THE MOT}

The laser cooling and trapping of $\mathrm{Rb}$ atoms cannot be easily set up as an undergraduate level experiment. The theoretical background and the operation of the equipment is too difficult to master in such a short time. Instead, the MOT served as a source of undergraduate research projects for physics major students who opted to do their graduate work at the Optoelectronics Lab. The students perform experiments to characterize the MOT, that is, to measure the physical quantities associated with the MOT, such as the number of trapped atoms, the lifetime of the trap, the volume and atomic density of the cloud of trapped atoms, and the temperature of the trapped atoms. Further, students investigate systematically the dependence of each of these quantities on the parameters of the MOT, such as the detuning of the trapping laser below resonance, the magnetic field of the antiHelmholtz coils, the intensity of the trapping and/or repumper laser beams, and the pressure inside the vacuum chamber.

Since the first time the MOT was used by students for their undergraduate research projects, two scientific research papers have been presented to the Philippine National Physics Congress, in 1999 and in 2000, and published in the conference proceedings. 9,10

\subsection{Numberof trapped atoms}

To determine the number of trapped atoms, the intensity of the fluorescence due to the trapped atoms was measured. A calibrated power meter head (Thorlabs ${ }^{\mathrm{TM}}$ S20MM Silicon Power Meter connected to a Fluke ${ }^{\mathrm{TM}} 45$ Dual Display Bench Multimeter) with an active detection diameter of $0.8 \mathrm{~cm}$ was placed near the glass cell, facing the center of the MOT. A convex lens with a diameter of $2.5 \mathrm{~cm}$ was placed between the MOT and the photodiode $5.0 \mathrm{~cm}$ from the center of the trap to produce a parallel beam Experiments were performed with the room darkened. A total of 10 measurements of 
the fluorescence with the trap on and off, at a given detuning of the trapping laser, were taken. The recorded differences in the digital voltmeter (DVM) readings when the trap is on and off were then averaged.

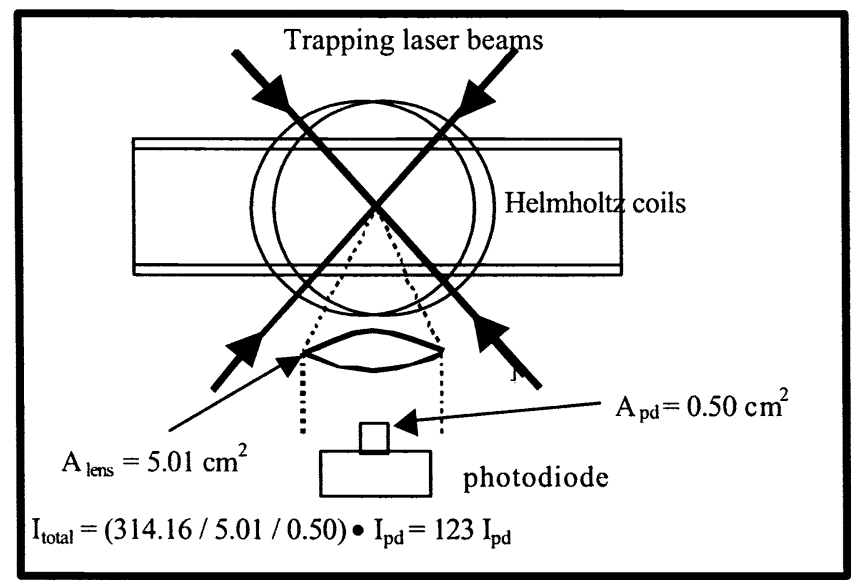

Figure 5. Measurement setup for determining the number of trapped atoms.

The number of trapped atoms is determined by the equation

$$
N=\frac{T_{L}}{R}
$$

where $T_{L}$ is the total amount of light scattered by the trapped $\mathrm{Rb}$ atoms (in units of photons/second) and $R$ (in units of photons/second) is the total amount of light scattered by one atom. The rate $R$ at which one atom scatters photons is given by the relation ${ }^{11}$

$$
R=\frac{\left(I / I_{s}\right) \pi \Gamma}{1+\left(I / I_{s}\right)+4(\Delta / \Gamma)^{2}}
$$

where $I=9.41 \mathrm{~mW} / \mathrm{cm}^{2}$ is the sum of the intensities of the six trapping beams, $\Gamma=5.9 \mathrm{MHz}$ is the natural linewidth of the ${ }^{87} \mathrm{Rb}$ transition, $\Delta=-16,-26$ or $-37 \mathrm{MHz}$ are the detunings of the trapping laser resonance, and $I_{S}=1.6 \mathrm{~mW} / \mathrm{cm}^{2}$ is the saturation intensity. Using the above data, $R$ is $3.1 \times 10^{6}, 1.3 \times 10^{6}$ and $6.8 \times 10^{5}$ photons/second atom, respectively, for the specified detunings. Table 1 shows the results of our measurements of the number of trapped ${ }^{87} \mathrm{Rb}$ atoms.

\begin{tabular}{|c|c|c|c|c|}
\hline$\Delta(M 1 / n)$ & $\begin{array}{l}\text { IPW } \\
(\mathrm{FWW})\end{array}$ & $\begin{array}{l}\text { Tromal } \\
(\mu w)\end{array}$ & $\begin{array}{l}\text { No. or } \\
\text { phroronsistec }\end{array}$ & $\begin{array}{l}\text { No. or } \\
\text { Arovis }\end{array}$ \\
\hline-16 & $5.9(4)$ & $3.7(2)$ & $1.5 \times 10^{13}$ & $4.7 \times 10^{6}$ \\
\hline-26 & $10.1(4)$ & $6.3(2)$ & $2.5 \times 10^{13}$ & $1.9 \times 10^{7}$ \\
\hline-37 & $14.2(4)$ & $8.9(3)$ & $3.5 \times 10^{13}$ & $5.1 \times 10^{7}$ \\
\hline
\end{tabular}

Table 1. Measurements of the number of trapped atoms 
The lifetime of the MOT is defined as the average time an atom remains trapped before it is removed (or escapes) from the trap by a collision with other atoms or through other mechanisms. This quantity is obtained by measuring the time it takes to load the MOT from zero atoms (trap off) to the steady-state number of atoms (trap on). A charge-coupled device (CCD) camera was used to measure the fluorescence of the trapped atoms, which is directly related to the number of trapped atoms.

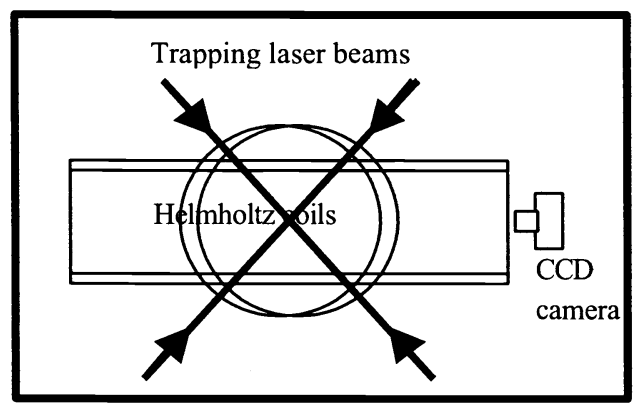

Figure 6. Setup for determining the lifetime of the MOT.

Initially the magnetic field in the MOT was switched off, disabling the trap without affecting the background fluorescence from the untrapped $\mathrm{Rb}$ atoms. Images were grabbed from the $\mathrm{CCD}$ camera with an image acquisition board (IMAQ, National Instruments ${ }^{\mathrm{TM}}$ ) and stored on a personal computer. The CCD image of the MOT was kept as small as possible yet large enough to see the whole cloud of trapped atoms so as to limit the image size, thereby allowing fast sampling. After starting the image capture program, the magnetic field was switched on, producing the cloud of the trapped atoms. A total of 100 images were grabbed from trap off to trap on, showing the growth of the Rb cloud. The result was then fitted to a single exponential.

For each data set of 100 images a total of 10 trials was taken. We reported an average lifetime for the MOT of $0.84(6) \mathrm{s}$, which fit well with the lifetime values reported by Wieman et al. The perfect single exponential growth is a signature that the sole mechanism that determines the trap lifetime is the removal of trapped atoms by collisions with background atoms. Reducing the background pressure by one order of magnitude is expected to extend the lifetime by a factor of 5 .

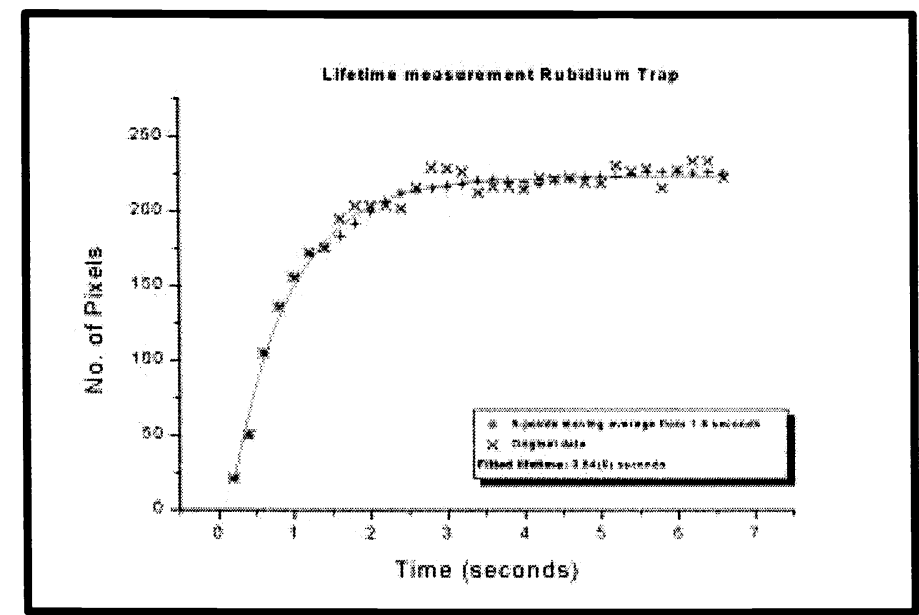

Figure 7. Exponential growth curve for our Rb MOT recorded at a sampling time of $200 \mathrm{~ms}$. 
Since our initial measurements of the lifetime of the trap, students doing the experiments for their research projects have been successfully measuring higher values of the MOT lifetime. The student project work in March 2000 reported a lifetime of $2.3 \pm 0.1 \mathrm{~s}$, while the March 2001 student research group reported an optimum lifetime of $2.7 \pm 0.6 \mathrm{~s}$. We attribute this increase in the measured values of the trap lifetime to the decrease in the pressure of the MOT vacuum system, from $10^{-8} \mathrm{mbar}$ in 1998 to almost $10^{-9} \mathrm{mbar}$ in 2001 .

\subsection{The volume and the atomic density of the MOT}

To obtain the atomic density of the MOT, the volume of the cloud of trapped $\mathrm{Rb}$ atoms was first determined. In three dimensions, the volume of the cloud of trapped atoms appears to be spherical, but actually assumes an ellipsoidal shape due to the quadrupole magnetic field configuration for the MOT. ${ }^{12}$ The magnetic field strength produced by the Helmholtz coils in the $z$-direction, which coincides with the axis of the coils, is twice as high as compared to the $x$ - and $y$-direction.

The six trapping laser beams, which overlap the center of the MOT, have a diameter of approximately $1 \mathrm{~cm}$. Within this volume the magnetic field contours are still perfect ellipsoids. The volume of an ellipsoid is given by $V=4 / 3 \cdot \pi \cdot a \cdot b \cdot c$, where $a, b$, and $c$ are the arms of the ellipsoid. For the calculation of the volume of the trapped atoms, we consider the following dimensions: Along the $z$-axis the arm was taken as $0.5 \mathrm{~cm}$. Along the $x$-and $y$-axis, the arms were taken to be $1 / \sqrt{2} \cdot 0.5 \mathrm{~cm}$. Therefore, the volume of trapped atoms was $V=0.26 \mathrm{~cm}^{3}$. The atomic density of our MOT is $2.0 \times 10^{8}$ atoms $/ \mathrm{cm}^{3}$ for a detuning of $-37 \mathrm{MHz}$.

\subsection{Temperature of trapped atoms}

For measuring atomic temperatures, the time -of-flight (TOF) method is a standard experiment. ${ }^{3}$ Using the TOF method, a probe laser beam is sent through the trapping cell at a distance of $1 \mathrm{~cm}$ below the atomic cloud. The probe beam enters a photodetector connected via an image acquisition (NI-IMAQ ${ }^{\mathrm{TM}}$ ) card to a computer. The expansion of the cloud when the trapping laser beams and the current are switched off simultaneously was monitored. Since this simultaneous switching requires sub-millisecond accuracy, a fast relay switch with a razor blade was designed to block the trapping laser beam electronically using LabVIEW ${ }^{\mathrm{TM}}$. A program that switches on the relay to block the trapping beams and at the same time switches off the current through the anti-Helmholtz coils was designed. Switching times of less than a millisecond have been measured for our relay, which makes it an inexpensive alternative to an optomechanical shutter. However, we have so far not achieved a temperature measurement using a photodetector.

Instead of measuring the TOF signal after blocking the trapping laser beams, we measured the expansion in optical molasses of the atomic cloud. In this method, the expansion of the cloud is monitored when the current through the anti-Helmholtz coils is switched off, but the trapping and repumper lasers are kept on. When the atomic cloud expands in optical molasses, the velocities will be slower due to damping. Therefore, the time scales involved are much longer than for standard TOF signals. This makes it possible to use a CCD camera for our experiment. Images of the expanding cloud were captured by a CCD camera connected to a computer with the video capture software VidCap ${ }^{\mathrm{T} M}$. Using a LabVIEW ${ }^{\mathrm{TM}}$ program to electronically switch off the current through the anti-Helmholtz coils, we observed that the cloud expanded in a direction to the upper left side of the CCD camera's field of view. This upward motion has been attributed to a residual magnetic field, including that of the earth's magnetic field and that of the ion pump for the MOT vacuum chamber. To compensate for this residual field, three pairs of Helmholtz coils were set up around the MOT, with their minimum separations limited by the MOT dimensions. For each Helmholtz pair, the current flows in the same direction to produce a 1-D magnetic field that is uniform at the center of the MOT. The current through each of the three pairs was adjusted until we obtained the optimum compensation, which shows the atomic cloud expanding uniformly in all directions.

We have to know the properties of our molasses in order for us to calculate the atomic velocity without damping, hence the need for a mathematical model in order to describe the expansion of an atomic cloud in optical molasses. This model uses a statistical description, in which the expansion of the cloud in optical molasses is treated as a diffusion process. Based on this model, the calculated temperature based on experimental measurements is always greater than the Doppler limit $144 \mu \mathrm{K}$ for $\mathrm{Rb}$. 
Making use of the statistical model, the net force on a particle, in this case an $\mathrm{Rb}$ atom undergoing a diffusion process is given by the relation ${ }^{13}$

$$
m \frac{d v}{d t}=-\alpha v+F(t)
$$

where $\alpha$ is known as the friction constant (hence the term $\alpha v$ is known as the frictional force) and $F(t)$ is the collisional force between atoms, which is a fluctuating force whose mean value vanishes. For the limiting case in which $t>>\gamma^{-1}$, where $t$ is the expansion time of the cloud of trapped $\mathrm{Rb}$ atoms and $\gamma^{-1}$ is the characteristic time constant of the system, given by the definition

$$
\gamma \equiv \frac{\alpha}{m}
$$

For the system of an expanding cloud of $\mathrm{Rb}$ atoms in optical molasses, the expansion time $t$ is of the order of several tens of $\mathrm{ms}\left(10^{-2} \mathrm{~s}\right)$, while the characteristic time constant $\gamma^{-1}$ for equation $(8)$ is of the order of $10^{-5} \mathrm{~s}$. The cloud expansion can then be described by the relation

$$
\left\langle x^{2}\right\rangle=\frac{2 k T}{\alpha} t
$$

where $T$ is the average temperature, $k$ is Boltzmann's constant, and $\left\langle x^{2}\right\rangle$ shows that a $\mathrm{Rb}$ atom moving through optical molasses behaves like a diffusing particle executing a random walk, since $\left\langle x^{2}\right\rangle$ is proportional to the expansion time $t$, and is therefore related to the radius (FWHM) of the expanding cloud. Taking a temporal plot of the measured FWHM of the radius of the cloud, the slope of the plot is then equal to $2 k T / \alpha$. From equations (2) and (7), it follows that the friction constant is given approximately by the relation

$$
\alpha \cong \hbar k^{2} \frac{\frac{2 I}{I_{S}} \cdot \frac{2 \Delta}{\Gamma}}{\left[1+\left(\frac{2 \Delta}{\Gamma}\right)^{2}\right]^{2}}
$$

Figure 8 shows the experimental setup for the temperature measurement. The vertical laser beam is parallel to the axis of the two anti-Helmholtz coils.

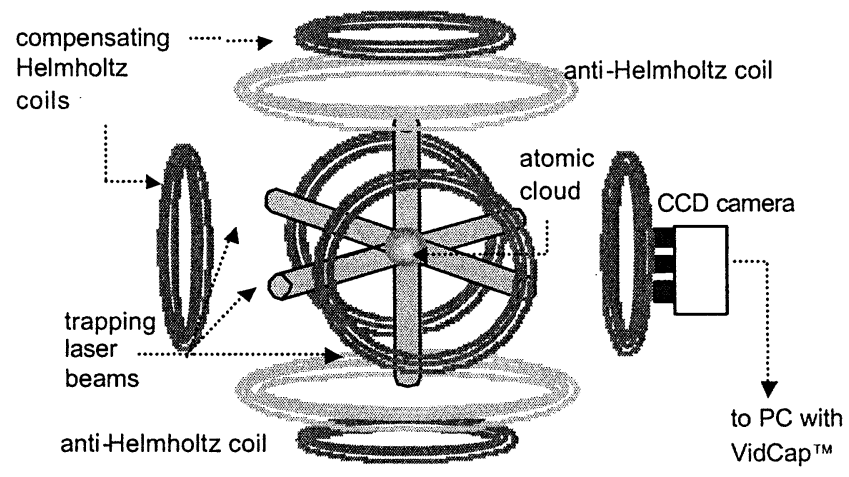

Figure 8 The experimental setup for the measurement of the temperature for atoms expanding in optical molasses, that is, when the current through the anti-Helmholtz coils is switched off without switching off the rapping laser beams.

The temperature was directly calculated $\Delta$ using equation (9) for different detunings. The lowest temperature measured was $184 \mu \mathrm{K}$ at a detuning $\Delta$ of $-38 \mathrm{MHz}$. A student undergraduate project is in the works to continue the experiments for the temperature measurement. This research work will also investigate the dependence of the temperature on the intensity $I$ of the trapping laser beams. Figure 9 shows a typical expansion of the cloud of $\mathrm{Rb}$ atoms after switching off the Helmholtz magnetic field. 

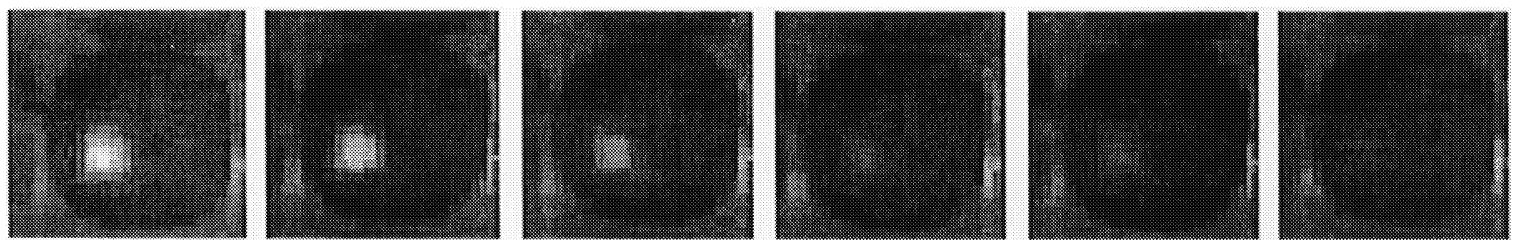

Figure 9. A frame-by-frame image at 26 frames/s of the cloud of trapped $\mathrm{Rb}$ atoms after switching off the current in the anti-Helmholtz coils, with corresponding compensation for residual magnetic fields.

\section{CONCLUSIONS AND FUTURE PLANS}

The presence of the MOT in our Optoelectronics Laboratory (OL) has encouraged interest in photonics research at San Carlos, and increased student interest in the field of optics and photonics. A number of undergraduate students who have undertaken their undergraduate project work at the OL have gone on to pursue graduate courses at the University of the Philippines (UP). Since 1997, the OL has established very strong academic and research linkages with the UP Photonics Laboratory. The active participation of USC-OL at the annual congress of Filipino physicists insures the continuity of academic and research activities in the field of laser cooling and trapping in the Philippines.

\section{ACKNOWLEDGMENTS}

The authors acknowledge the Netherlands University Foundation for International Cooperation (NUFFIC) for its financial support for this project, and to the faculty and staff of the Atomic and Laser Physics Group of the Vrije Universiteit Amsterdam, The Netherlands, for the scientific and technical guidance to get this work started at the University of San Carlos, Cebu City, Philippines, under the supervision of the Physics Development Project of NUFFIC.

\section{REFERENCES}

1. S. Chu, "The manipulation of neutral particles," Rev. Mod. Phys. 70, pp. 685-706 (1998).

2. C.N. Cohen-Tannoudji, "Manipulating atoms with photons," Rev. Mod. Phys. 70, pp. 707-719 (1998).

3. W.D. Phillips, "Laser cooling and trapping of neutral atoms," Rev. Mod. Phys. 70, pp. $721-741$ (1998).

4. W. Ketterle, "Experimental studies of Bose-Einstein condensation," Physics Today, pp. 30-35 (1999).

5. M.H. Anderson, J.R. Ensher, M.R. Matthews, C.E. Wieman, and E.A. Cornell, Science 269, p. 198 (1995).

6. W.D. Phillips, "Fundamental Systems in Quantum Optics," Les Houches Session LIII, Eds. J. Dalibard, J.Raimond, and J. Zinn-Justin, Elsevier (1990).

7. S.J.H. Petra, "Development of frequency-stabilized laser diodes for building a magneto-optical trap," M.S. thesis, Universiteit van Amsterdam, The Netherlands (1998).

8. W. Demtröder, Laser spectroscopy, basic concepts and instrumentation, $2^{\text {nd }}$ ed., Springer-Verlag, Berlin (1998).

9. J.W.F. Liwag, G.J. Kuik, and M. Daza, "Measurements of the trap lifetime and the number of trapped atoms in a magneto-optical trap (MOT) for ${ }^{87} \mathrm{Rb"}$ ", Proceedings of the $17^{\text {th }}$ SPP Philippine National Physics Congress, Tacloban City, Philippines, pp. 185-187 (1999).

10. J.W.F. Liwag et al, "Characterization of the magneto-optical trap for Rb atoms," Proceedings of the $18^{\text {th }}$ SPP Philippine National Physics Congress, Palawan, Philippines, pp. 214-216 (2000).

11. C. Wieman, G. Flowers, and S. Gilbert, "Inexpensive laser cooling and trapping for undergraduate laboratories," Am. J. Phys. 63, pp. 317-330 (1995).

12. T. Bergeman, G. Erez, and H. Metcalf, "Magnetostatic trapping fields for neutral atoms," Phys. Rev. A 35, pp. 1535-1546(1987).

13. F. Reif, Fundamentals of statistical and thermal physics, McGraw-Hill Kogakusha, Ltd., Tokyo, Japan, pp. 565567 (1981). 